\title{
Survival analysis of Electrical Power Distribution systems using Weibull Regression
}

\author{
Matheus de Souza Sant'Anna Fogliatto* \\ Talysson Manoel de Oliveira Santos* Michel Bessani** \\ Carlos Dias Maciel* \\ * Lab. Processamento de Sinais, Dep. Eng. Elétrica e Computação, \\ USP, São Carlos, Brasil (e-mail: matheusfogliatto@usp.br). \\ ** Graduate Program in Electrical Engineering - Federal University of \\ Minas Gerais - Av. Antônio Carlos 6627, 31270-901, Belo Horizonte, \\ MG, Brazil (e-mail: mbessani@eee.ufmg.br)
}

\begin{abstract}
Critical infrastructures are systems present in our society, which interfere in several ways when they do not operate in their normal conditions. Electric Power Distribution systems are an example of critical infrastructure, and therefore, the study of the common factors that affect their performance has great relevance. The concept of time to event (Lifetime Data Analysis) makes it possible to use survival analyses to estimate the influence of different dangers on the reliability of systems. A Weibull distribution was used to implement a Weibull regression, using climate data and information about failures of a Brazilian Electrical Power Distribution System, allowing verification of how climatic events impact this type of critical infrastructure. The results presented describes the failure chance given certain climatic conditions, indicating the effect of such exogenous covariates. With greater awareness of potential threats, the reliability of the system can be incresead by better planning and for threats mitigation.
\end{abstract}

Keywords: Electric Power Distribution system; Reliability Analysis; Climatic Events; Weibull Regression.

\section{INTRODUCTION}

Electric Power Distribution Systems (DSs) are a critical infrastructure of our society mainly because different activities depend on it (Brem, 2015). As DSs occupy large areas, they are exposed to various threats. The causes of failures on DSs (Abedi et al., 2019) are climatic events, such as high or low temperatures, strong wind, storms (Burillo et al., 2016), accidents like a collision with vehicles, animals or vegetation (Sahai and Pahwa, 2006; Radmer et al., 2002), technical errors as devices failures and load transfer ( $\mathrm{Gu}$ et al., 2017) and rare events like terrorism, vandalism and cyber attacks (Ni and Li, 2018). Protective measures against these threats are an object of interests for governments around the world (Setola et al., 2016).

Climatic events impact on DSs are important because of the significant contribution in the total of failure events (Złotecka and Sroka, 2018), and due to the influence of climate-changing (Sridharan et al., 2019), these events should increase in both, intensity and occurrence. Another point of interest is the extreme rare climatic events, that can generate massive blackouts (Liu and Zhong, 2017).

This study analyzes the influence of three climatic events in a real Electric Power Distribution system. Maximum Wind Speed, the number of atmospheric discharges and rain precipitation (Caswell et al., 2011) are the chosen events. A way to study the influence of climatic events on the reliability of DSs is the application of information about these events together with information about failure occurrence on statistical methods.

Statistical methods are used to model problems involving death or failure in different areas, such as Medicine (Cheng et al., 2018; Ferlay et al., 2015), Engineering (Luo et al., 2018), Agricultural and Biological Sciences (Shin et al., 2018) according to the arrange and the distribution that the data follows. For studies involving covariates, as climatic events, regressions are an alternative. Regression models can use different distributions and techniques options to adjust the best representation of the data intended to be used (Upadhya and Cheeran, 2018). Survival analysis is another approach applicable to regression methods, that usually uses time as the primary variable, and provides survival and hazard functions that are interesting to be analyzed. Both ways can be applied in DSs failure data associated with climatic events as covariates, as in Fanucchi et al. (2016) with a Negative Binomial Regression for failure rate and Bessani et al. (2016) with Survival Analysis using mainly non-parametric techniques.

Survival analysis, also known as reliability analysis for engineering, analyzes data that depend to an occurrence of an event (death of a patient, failure of equipment) due to a starting point (Abdelsalam et al., 2018). Survival analysis grew in importance after World War II, in studies about the reliability of military equipment (Singh and 
Mukhopadhyay, 2011). The option of Survival analyses have three main methods, non-parametric, parametric and semi-parametric, and can be applied in areas such as Medicine (Moolgavkar et al., 2018), Engineering (Dantas et al., 2010) and Reliability (Murthy et al., 2004). Parametric Statistics needs a type of probability distribution and can be more accurate than non-parametric techniques (Zhang, 2016).

On this paper, Weibull Distribution followed by a Weibull Accelerated Failure Time (AFT) Regression was used to make reliability analyze. Weibull is a parametric model that provides excellent results for observe covariates influence in the occurrence of the event of interest (Zhang, 2016). This distribution generates good results for survival studies, including life and fatigue testing (Murali et al., 2017) and can be applied in small samples (Abernethy, 2004). It has the three-parameters (Yang and Jiang, 2019) and two-parameters form (Hubbard et al., 2011), that it was the one that was used here.

The construction of a Weibull model for reliability analysis for a DSs associated with climatic events is very interesting for reliability engineering because time to event models is necessary to estimation of reliability indicators such as System Average Interruption Duration Index (SAIDI), System Average Interruption Frequency Index (FAIDI) (Alvehag and Söder, 2011) and resilience indices (Bessani et al., 2018; Panteli et al., 2017). This model can be useful for decision making in the electric power distribution system management, such as maintenance crew allocation, reducing risks and costs improving the performance of the system and studies for contingencies plans (Menon et al., 2019).

In Section 2, the statistical theory is presented, and in Section 3, a description of the data used is presented, and how they were processed to perform the proposed analysis and describe the computational tools used to analyze the data and obtain the model. In Section 4 the results generated by applying survival analyse in the database are shown. For last, in Section 5, a discussion is made about the results and its interpretations, and the conclusions and future improvements are presented.

\section{STATISTICAL MODELING}

For survival analysis, some concepts are very usual and should be defined. "Time" can be interpreted as the time until an event of interest occurs (failure of equipment, death of a patient). "Event" is the occurrence (or not) of the event of interest (a failure happened, a patient died).

"Censored observation" is the objects of study that left the study or did not have the occurrence of the event until the end of the study. For this type of data, survival time will not be very accurate, so it's important to identify them to make good modeling.

Graphics are the main output of these models, and three functions are very usual. The "survival function", also known as "reliability function", is the probability that a subject survives longer that time t, "Hazard function" is the event rate at time $t$ conditional on survival until time $t$ or later $(\mathrm{S}(\mathrm{t})=\mathrm{P}(\mathrm{T} \geq \mathrm{t}))$ and "Cumulative hazard function" is the accumulation of the hazard over time. Cumulative hazard is a rate, so it is not restricted to the interval $[0$; 1].

Generally, with "Pr" as probability, "t" as time and "T" a random variable denoting the time of death or Failure, the survival $(S(t))$ or reliability $(R(t))$ function can be defined as:

$$
S(t)=R(t)=\operatorname{Pr}(T>t)
$$

The lifetime distribution function $(F)$ is defined as the complement of the reliability function:

$$
F(t)=\operatorname{Pr}(T \leq t)=1-R(t)
$$

The density function of the lifetime distribution represents the rate of death or failure events per unit time and is defined as:

$$
f(t)=\frac{d}{d t} F(t)
$$

Making relations of the previous functions:

$$
R(t)=\operatorname{Pr}(T>t)=\int_{t}^{\infty} f(u) d u=1-F(t)
$$

The Hazard function can be described as:

$$
h(t)=\lim _{d t \rightarrow 0} \frac{\operatorname{Pr}(t \leq T<t+d t)}{d t R(t)}=\frac{f(t)}{R(t)}=-\frac{R^{\prime}(t)}{R(t)}
$$

and the Cumulative Hazard function:

$$
H(t)=-\log R(t)=\int_{0}^{t} h(u) d u
$$

and:

$$
R(t)=\exp [-H(t)]=1-F(t), t>0
$$

In this paper, Weibull distribution was used. This distribution has an important characteristic that is his failure rate. It can be constant, with a growing or with a decreasing form. Weibull probability density function for a random variable $\mathrm{T}$ is shown below:

$$
f(t)=\frac{\rho}{\lambda^{\rho}} t^{\rho-1} \exp -\left(\frac{t}{\lambda}\right)^{\rho}, t \geq 0
$$

The shape parameter and the scale parameter are respectively $\rho$ and $\lambda$.

The reliability function can be obtained as:

$$
R(t)=\exp \left(-\left(\frac{t}{\lambda}\right)^{\rho}\right), \lambda>0, \rho>0
$$

And the hazard and cumulative hazard function are:

$$
h(t)=\frac{\rho}{\lambda}\left(\frac{t}{\lambda}\right)^{\rho-1}
$$




$$
H(t)=\left(\frac{t}{\lambda}\right)^{\rho}
$$

For AFT models, we have a comparison between two reliability functions, defined as $R_{A}(t)$ and $R_{B}(t)$ in equation (12), related by a accelerated failure rate, called $\lambda$ (the same $\lambda$ present in Weibull Distribution):

$$
R_{A}(t)=R_{B}\left(\frac{t}{\lambda}\right)
$$

More generally, we can model $\lambda$ as a function of covariates $b_{i}$, being $b_{0}$ the intercept:

$$
\begin{gathered}
R_{A}(t)=R_{B}\left(\frac{t}{\lambda(x)}\right) \\
\lambda(x)=\exp \left(b_{0}+\sum_{i=1}^{n} b_{i} x_{i}\right)
\end{gathered}
$$

As can be seen in equation (14), the covariates can accelerate or decelerate the failure time. A unit increase in $x_{i}$ means the average/median survival time changes by a factor of $\exp \left(b_{i}\right)$.

Defining reliability function by:

$$
R(t ; x, y)=\exp \left(-\left(\frac{t}{\lambda(x)}\right)^{\rho(y)}\right)
$$

And Cumulative hazard function is given by:

$$
H(t ; x, y)=\left(\frac{t}{\lambda(x)}\right)^{\rho(y)}
$$

A more detailed explanation can be found in survival analysis books, as Colosimo and Giolo (2006), or in the documentation of Lifelines Package.

\section{METHODS}

\subsection{Data description}

Two datasets are used in this paper. The first one describes events of failure on a power distribution system composed of 24 distribution feeders, from 01/01/2012 to $31 / 12 / 2014$, which include the date and hour of each failure. Failures can be defined as a power interruption of any duration. In this dataset, 323 different causes of failure are identified, with causes associated with maintenance of the network, accidents with animals such as insects and birds, climatic events or faults with a not identified cause. The second database provides daily information about climate events for the same period, such as the daily maximum wind speed in kilometer per hour, amount of rain (volume of precipitation) by millimeter and number of atmospheric discharges.

The system of this work, presented in Fig. 1, are from a midsize Brazilian city, founded just over 80 years ago. So, is expected that the Distribution System is relatively new, and, therefore, is robust against most common threats.

For each failure, it was considered that the system goes back to work fully instantly, as in his initial state, that is, failures are assumed to be independent of each other

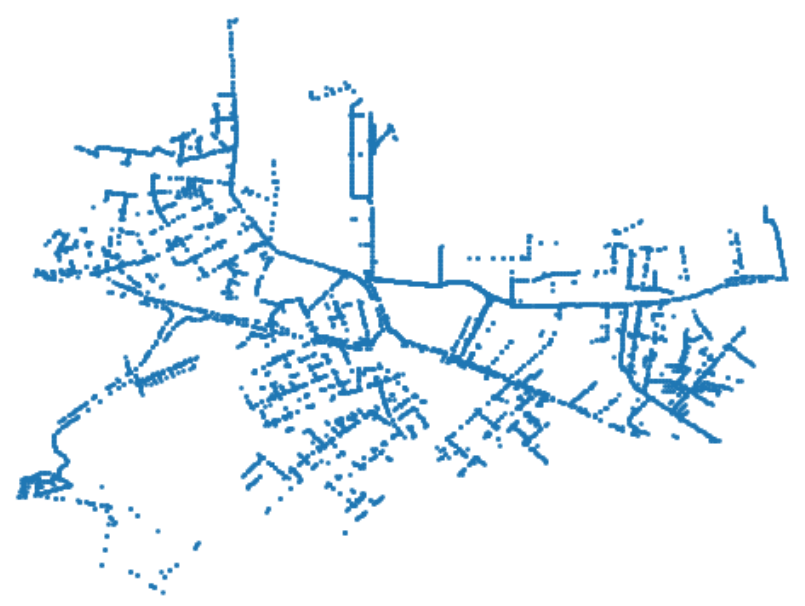

Figure 1. Graph representation of a brazilian Electric Power Distribution System, composed of 3536 nodes, 3254 edges.

Table 1. Example of how Survival Time was obtained

\begin{tabular}{ccc}
\hline Initial Date/Hour & Failure Date/Hour & Survival Time (Min) \\
\hline 01-01-2012 00:00:00 & 02-01-2012 07:31:00 & 1891 \\
02-01-2012 07:31:00 & 02-01-2012 14:29:00 & 418 \\
02-01-2012 14:29:00 & 03-01-2012 15:34:00 & 1505
\end{tabular}

(Xiaohui et al., 2016). So, making a parallel with medical applications, the time-to-failure (time to event) is the lifetime, and each event (failure occurrence) can be interpreted as the death of a subject. The minimum value registered of a lifetime in the database was 1 minute, and the maximum was 8782 minutes (a little more than six days).

Making '01/01/2012 00:00:00' as the starting time, the lifetime of our system until each failure was calculated. After a failure, the failure time will be the new initial time. For easy understanding, the survival time can be obtained as in Tab. 1. The number of 2261 failures was observed in the proposed period. Fig. 2 shows a histogram of the survival duration times, in logarithmic scale.

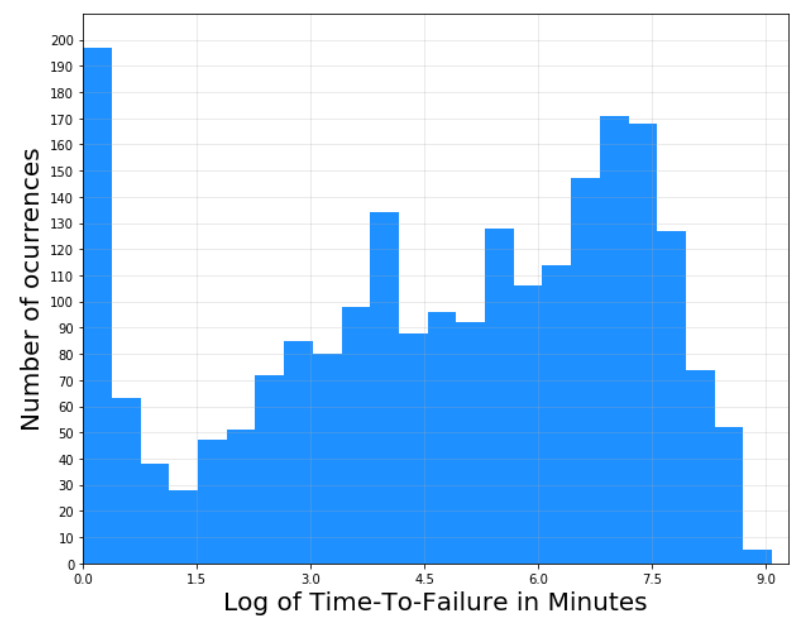

Figure 2. Histogram of the survival time of the system

A new database was created with information of the time to failure (how much time the system have not failed) in 
the scale of minutes, related with the values for the climatic events in the day that each failure happened, as in Tab. 2.

\subsection{Computational environment}

For this study, with Ubuntu version 18.04.2 LTS, Python version 3.6.7 was used on Jupyter Notebook version 5.6.0. Pandas Package version 0.24.2 was used to open and manage the databases, Lifelines Package version 0.20.1 was used for the statistics, Matplotlib Package version 3.0.3 was used to figure plotting and Networkx Package version 2.2 for analyze the size of the used system.

\section{RESULTS}

Using the database illustrated in Tab. 2, we set the columns (main variables) that represents "Duration" and "Event". In this table, the columns "Survival Time (Minutes)" and "Event" represent these two variables, respectively. As the focus of this study is power systems failures, for the "Event" column, there is no presence of censored observations (so, for all observations, the system failed).

First, a Weibull fitting was used, utilizing Time and Event as variables. The estimated parameters that defines the distribution are shown on Tab. 3 .

With the distribution defined, the three principal graphs from this method can be obtained. In Fig. 3 and 4, Reliability, Hazard and Cumulative Hazard functions and the confidences intervals of each one are presented.

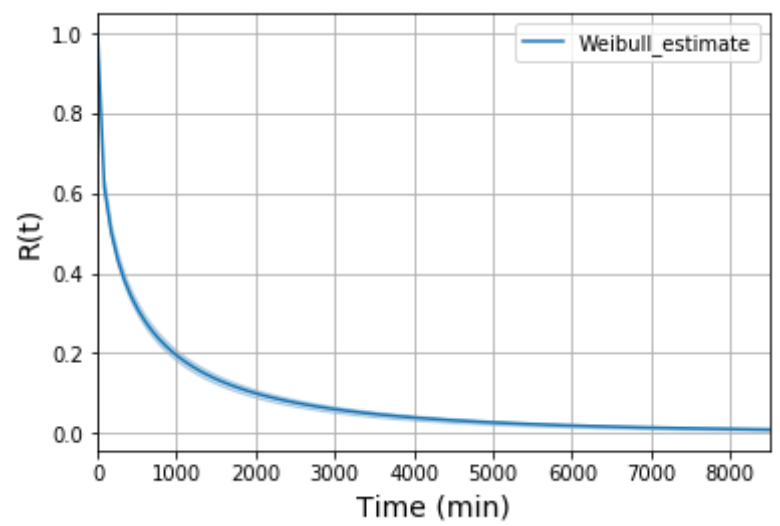

Figure 3. Reliability Function $(\mathrm{R}(\mathrm{t}))$ with Weibull Fitting

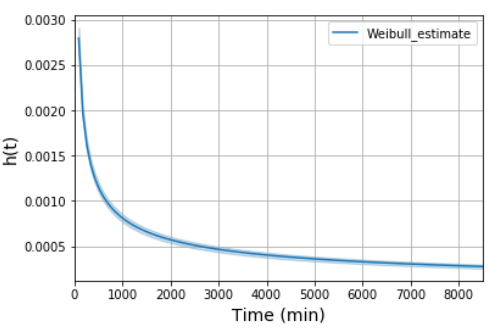

(a)

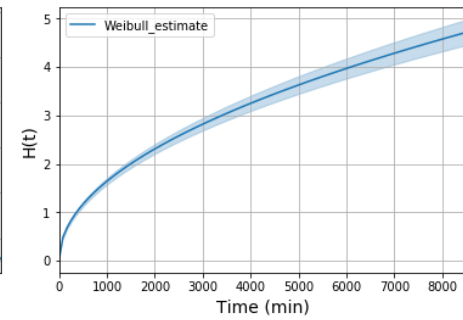

(b)
Figure 4. Weibull Fitting: a) Hazard Function and b) Cumulative Hazard Function.

To include covariates, Weibull Regression (Weibull AFT Model) were used, and the obtained parameters are presented in Tab. 4. In Fig. 5 and 6, reliability functions with

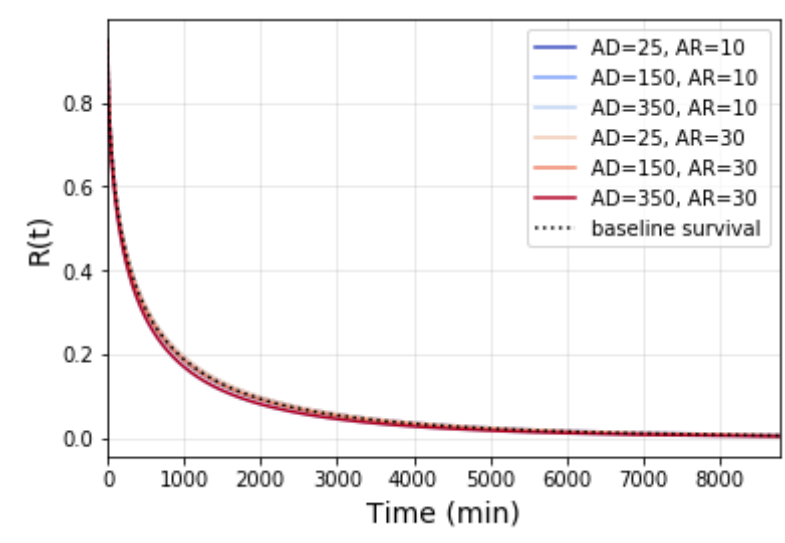

Figure 5. Reliability Function $(\mathrm{R}(\mathrm{t}))$ of the power system for different values of atmospheric discharges (AD) and Amount of Rain (AR, in Millimeters)

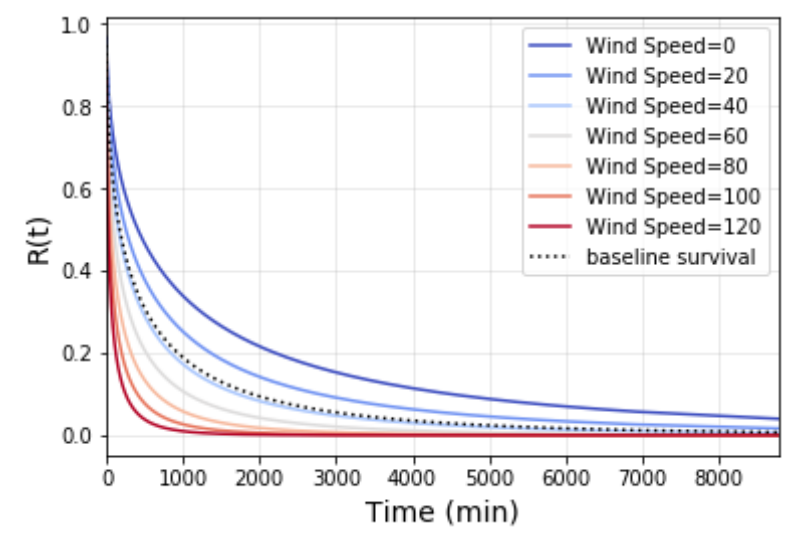

Figure 6. Reliability Function $(\mathrm{R}(\mathrm{t}))$ of the power system for different values of maximum wind speed in kilometer/hour

different values for each variable (Number of thunders, Maximum Wind Speed and amount of Rain) are plotted with the baseline reliability function curve (from the AFT model).

\section{DISCUSSION AND CONCLUSION}

Studies in reliability engineering, applied in power systems, have a strong correlation with climatic events and the concept of resilience, and both are very related with time. So, the development of a model using time, as in survival analyses, can be more interesting than the approach using count data, due to a more explicit link with those crucial concepts.

The Weibull distribution was chosen due to its flexibility and because this is one of the most used distributions in survival analysis. Regression makes it possible to evaluate the baseline survival and the influence of covariates in a real distribution power system.

Our three variables, number of thunders, maximum wind speed in kilometer/hour and amount of rain in millimeters, as the intercept, that is the intrinsic coefficient for the lifetime of the system (presented in Tab. 4), can be compared by the coefficient values and "p-value". Generally, if $p \leq 0.05$, we can say our result is not statistically 
Table 2. Samples of the Database used to apply both Weibull Fitter and Weibull AFT Fitter, that describes the values of the climate events and the survival time of the system of each failure event

\begin{tabular}{cccccc}
\hline Date & Number of Thunders & Wind Speed $(\mathbf{K m} / \mathbf{h})$ & Rain $\mathbf{( m m})$ & Survival Time (Minutes) & Event \\
\hline $2012-01-01$ & 1.0 & 30.0 & 0.0 & 1891 & 1 \\
$2012-01-02$ & 23.0 & 45.0 & 34.0 & 418 & 1 \\
$2012-01-02$ & 23.0 & 45.0 & 34.0 & 1505 & 1
\end{tabular}

Table 3. Weibull Fitting Coeficients

\begin{tabular}{ccccc}
\hline & Coef. & Lower 0.95 & Upper 0.95 & p-value \\
\hline$\widehat{\lambda}$ & 369.23272 & 336.78244 & 401.68299 & $<5 \mathrm{e}-06$ \\
$\hat{\rho}$ & 0.49446 & 0.47810 & 0.51082 & $<5 \mathrm{e}-06$ \\
\hline
\end{tabular}

Table 4. Weibull AFT Regression Coeficients

\begin{tabular}{cccccc}
\hline & & Coef. & Lower 0.95 & Upper 0.95 & p-value \\
\hline$\widehat{\lambda}$ & Intercept & 6.78231 & 6.55330 & 7.01131 & $<5 \mathrm{e}-06$ \\
& Thunder & -0.00048 & -0.00097 & 0.00002 & 0.05870 \\
& Wind & -0.02409 & -0.03048 & -0.01770 & $<5 \mathrm{e}-06$ \\
& Rain & 0.00061 & -0.00569 & 0.00691 & 0.84956 \\
$\widehat{\rho}$ & Intercept & -0.68937 & -0.72234 & -0.65640 & $<5 \mathrm{e}-06$ \\
\hline
\end{tabular}

significant. So, from our results, we can analyze the three climate events:

First, rain, with a positive value of 0.00061 , that could be interpreted as more amount of rain could help the system survive more time (but his confidence interval varies from a negative value to a positive value), has a small coefficient and an $p \geq 0.05$, that could demonstrate a minor influence in the survival time.

A coefficient of -0.00048 was obtained for thunder. Analysing p-value and the confidence interval, the number of atmospheric discharges has a low influence on the developed failure model, but may be considered.

For last, wind covariate has a coefficient of -0.02409 , the confidence interval is only negative and has a $p \leq 0.05$. So, from the three covariates included in this model, the wind was the most impacting climate variable and the only one which resulted in a statistically significant regression coefficient.

Comparing Fig. 5 and 6 , we could see the influence of each variable. Analyzing Rain and Thunder covariates, by the $p$-values $\geq 0.05$ and the confidence interval passes through 0 , we can conclude that those covariates do not present statistical significance. For those figures, we used the variation range present in the database, excluding isolated events (for example, a day with 2267 atmospheric discharges or a day with a maximum wind speed of $104 \mathrm{Km} / \mathrm{h}$ ). The baseline survival curve is equal to the predicted survival curve at all average values (the mean values for the number of atmospheric discharges, the maximum wind speed and the amount of rain are 75.1415 , 35.6850 and 7.0623, respectively), and has a survival time of 172 minutes for survival function equal to 0.5.

With the results of this paper, we estimate the relevance of different climatic events on the occurrence of failures of Electric Power Distribution systems. Using a parametric model as Weibull with a good fitting to the data and estimate the impact of three of the main factors associated to failure events in DSs are the improvements when comparing with others researches.
The knowledge of which hazard is more dangerous helps reliability engineer to search for improvements for a specific characteristic, for the different equipment's that make up the system, to amplify robustness. Another application is to use climate forecasting together with the model proposed to simulation frameworks, making possible contingencies plans and an optimal maintenance crew allocation to areas more vulnerable to the occurrence of a failure event. For future implementations, we can add more feeders (making a model with a database from a more significant area), that should change the coefficients and the statistical significance of each climate event, creating a better model. More variables can be used, such as other climatic events. Another point could be to use multiplicative effects of covariates.

\section{AGRADECIMENTOS}

This work was partialy suport by FAPESP: 2014/50851-0 e 2018/19150-6; CNPq: 465755/2014-3; Copel: PD2866$0504 / 2018$.

\section{REFERENCES}

Abdelsalam, N., Elbashir, M., and Saadeldeen, S. (2018). Applying cox regression in time to event data. 2018 International Conference on Computer, Control, Electrical, and Electronics Engineering, ICCCEEE 2018.

Abedi, A., Gaudard, L., and Romerio, F. (2019). Review of major approaches to analyze vulnerability in power system. Reliability Engineering and System Safety, 183, $153-172$.

Abernethy, R.B. (2004). The New Weibull handbook: reliability and statistical analysis for predicting life, safety, supportability, risk, cost and warranty claims; 5th ed. Dr. Robert B. Abernethy, North Palm Beach, FL.

Alvehag, K. and Söder, L. (2011). A reliability model for distribution systems incorporating seasonal variations in severe weather. IEEE Transactions on Power Delivery, 26(2), 910-919.

Bessani, M., Fanucchi, R.Z., Achcar, J.A., and Maciel, C.D. (2016). A statistical analysis and modeling of repair data from a brazilian power distribution system. In 2016 17th International Conference on Harmonics and Quality of Power (ICHQP).

Bessani, M., Massignan, J.A.D., Fanucchi, R.Z., Camillo, M.H.M., London, J.B.A., Delbem, A.C.B., and Maciel, C.D. (2018). Probabilistic assessment of power distribution systems resilience under extreme weather. IEEE Systems Journal, 1-10.

Brem, S. (2015). Critical infrastructure protection from a national perspective. European Journal of Risk Regulation, 6(2).

Burillo, D., Chester, M., and Ruddell, B. (2016). Electric grid vulnerabilities to rising air temperatures in arizona. Procedia Engineering, 145, 1346-1353. 
Caswell, H., Forte, V., Fraser, J., Pahwa, A., Short, T., Thatcher, M., and Werner, V. (2011). Weather normalization of reliability indices. IEEE Transactions on Power Delivery, 26(2), 1273-1279.

Cheng, Q., Wei, T., Jia, Y., Farbiak, L., Zhou, K., Zhang, S., Wei, Y., Zhu, H., and Siegwart, D. (2018). Dendrimer-based lipid nanoparticles deliver therapeutic fah mrna to normalize liver function and extend survival in a mouse model of hepatorenal tyrosinemia type i. Advanced Materials, 30(52).

Colosimo, E. and Giolo, S. (2006). Análise de sobrevivência aplicada. Edgard Blücher.

Dantas, M., Valença, D., Platiny da Silva Freire, M., Medeiros, P., Da Silva, D., and Aloise, D. (2010). Weibull-regression models to study failure data in oil pumps. Produção, 20, 127-134.

Fanucchi, R.Z., Bessani, M., Camillo, M.H.M., London, J.B.A., and Maciel, C.D. (2016). Failure rate prediction under adverse weather conditions in an electric distribution system using negative binomial regression. In 2016 17th International Conference on Harmonics and Quality of Power (ICHQP).

Ferlay, J., Soerjomataram, I., Dikshit, R., Eser, S., Mathers, C., Rebelo, M., Parkin, D., Forman, D., and Bray, F. (2015). Cancer incidence and mortality worldwide: Sources, methods and major patterns in globocan 2012 International Journal of Cancer, 136(5), E359-E386.

Gu, T., Janssen, J., Tazelaar, E., and Popma, G. (2017). Risk prediction in distribution networks based on the relation between weather and (underground) component failure. CIRED - Open Access Proceedings Journal, (1), 1442-1445.

Hubbard, J., Stoudt, M., and Possolo, A. (2011). Topographic analysis and weibull regression for prediction of strain localisation in an automotive aluminium alloy. Materials Science and Technology, 27(7), 1206-1212.

Liu, Y. and Zhong, J. (2017). Risk assessment of power systems under extreme weather conditions - a review. 2017 IEEE Manchester PowerTech, Powertech $201 \%$.

Luo, H., Xu, D., and Bao, J. (2018). Outage capacity analysis of mimo system with survival probability. IEEE Communications Letters, 22(6), 1132-1135.

Menon, M., Chattopadhyay, G., and Beebe, R. (2019). Decision support tools for preventive maintenance intervals and replacement decisions of engineering assets. IEEE International Conference on Industrial Engineering and Engineering Management, 2019-December, 257-261.

Moolgavkar, S., Chang, E., Watson, H., and Lau, E. (2018). An assessment of the cox proportional hazards regression model for epidemiologic studies. Risk Analysis, 38(4), 777-794.

Murali, G., Vincy Monika, T., Suraj, K., Ramkumar, V., and Karthikeyan, K. (2017). Assessment of impact strength of fibre reinforced concrete by two parameter weibull distribution. ARPN Journal of Engineering and Applied Sciences, 12(5).

Murthy, D.P., Bulmer, M., and Eccleston, J.A. (2004). Weibull model selection for reliability modelling. Reliability Engineering IE System Safety, 86(3).

Ni, M. and Li, M. (2018). Reliability assessment of cyber physical power system considering communication failure in monitoring function. In 2018 International Conference on Power System Technology (POWERCON),
3010-3015.

Panteli, M., Pickering, C., Wilkinson, S., Dawson, R., and Mancarella, P. (2017). Power system resilience to extreme weather: Fragility modeling, probabilistic impact assessment, and adaptation measures. IEEE Transactions on Power Systems, 32(5), 3747-3757.

Radmer, D., Kuntz, P., Christie, R., Venkata, S., and Fletcher, R. (2002). Predicting vegetation-related failure rates for overhead distribution feeders. IEEE Transactions on Power Delivery, 17(4), 1170-1175.

Sahai, S. and Pahwa, A. (2006). A probabilistic approach for animal-caused outages in overhead distribution systems. 2006 9th International Conference on Probabilistic Methods Applied to Power Systems, PMAPS.

Setola, R., Luiijf, E., and Theocharidou, M. (2016). Critical Infrastructures, Protection and Resilience. Springer International Publishing, Cham.

Shin, H., Oh, S., Kim, D., Hong, J., Yun, J., Lee, S., and Son, K.H. (2018). Induced freezing tolerance and free amino acids perturbation of spinach by exogenous proline. Journal of Plant Biotechnology, 45, 357-363.

Singh, R. and Mukhopadhyay, K. (2011). Survival analysis in clinical trials: Basics and must know areas. Perspectives in Clinical Research, 2(4), 145-148.

Sridharan, V., Broad, O., Shivakumar, A., Howells, M., Boehlert, B., Groves, D.G., Rogner, H.H., Taliotis, C., Neumann, J.E., Strzepek, K.M., et al. (2019). Resilience of the eastern african electricity sector to climate driven changes in hydropower generation. Nature communications, 10(1), 302.

Upadhya, S. and Cheeran, A. (2018). Performance comparison of regression techniques in predicting parkinson disease severity score using speech features. Biomedical Engineering - Applications, Basis and Communications, $30(4)$.

Xiaohui, Y., Wuzhi, Z., Xinli, S., Guoyang, W., Tao, L., and Zhida, S. (2016). Review on power system cascading failure thoeries and studies. 2016 International Conference on Probabilistic Methods Applied to Power Systems, PMAPS 2016 - Proceedings.

Yang, C.W. and Jiang, S.J. (2019). Weibull statistical analysis of strength fluctuation for failure prediction and structural durability of friction stirwelded al-cu dissimilar joints correlated to metallurgical bonded characteristics. Materials, 12(2).

Zhang, Z. (2016). Parametric regression model for survival data: Weibull regression model as an example. Annals of Translational Medicine, 4(24).

Złotecka, D. and Sroka, K. (2018). The characteristics and main causes of power system failures basing on the analysis of previous blackouts in the world. 2018 International Interdisciplinary PhD Workshop, IIPhDW 2018, 257-262. 